\title{
INVESTIGATION OF WIRE MOTION IN SUPERCONDUCTING MAGNETS*
}

\author{
A. Devred \\ Superconducting Super Collider Laboratory ${ }^{\dagger}$ \\ 2550 Beckleymeade Ave. \\ Dallas, TX 75237
}

September 1990

\section{DISCLAIMER}

\begin{abstract}
This 1cport was prepared as an account of work sponsored by an agency of the I/nit ${ }^{*}$ d States Government. Neither the United States Government nor any agency thereof, nor any of their employees, makes any warranty, express or implied, or assumes any legal liability or responsibility for the accuracy, completeness, or usefulness of any information, apparatus, product, or process disclosed, ot represents that its use would not infringe privately owned rights. Reference herein to ariy specific commercial product, process, or service by trade name, trademark, manufacturer, or otherwise does not necessarily constitute or imply its endorsement, recommendation, or favoring by the United States Government or any agency thereof. The views and opinions of authors expressed herein do not necessarily state or reflect those of the United States Government or any agency thereof.
\end{abstract}

"Presented at the Applied Superconductivity Conference, Snowmass, Colorado, September 24-28, 1990.

†Operated by the Universities Research Association, Inc., for the U.S. Department of Energy under Contract No. DE-AC02-89ER40486. 


\author{
T. Ogitsu and $K$. Tsuchiva \\ KEK, National Laboratory for High Energy Physics, \\ 1.1 Oho, Tsukuba-shi, \\ Ibaraki-ken 305 Japan \\ A. Devred \\ KEK, National Laboratory \\ for High Energy Physics, Japan \\ \& SSC Laboratory ${ }^{\dagger}$ \\ 2550 Beckleymeade Avenue \\ Dallas, TX 75237 USA
}

\begin{abstract}
The large Lorentz forces occuring during the excitation of superconducting magnets can provoke sudden motions of wire, which eventually release enough energy to trigger a quench. These wire motions are accompanied by two electromagnetic effects: an induced emf along the moved wire, and a local change in flux caused by the minute dislocation of current. Both effects cause spikes in the coil voltage. Voltage data recorded during the excitation of a superconducting quadrupole magnet which clearly exhibit such events are here reported. Interpretations of the voltage spikes in terms of energy release are also presented, leading to insights on the spectrum of the disturbances which occur in real magnets.

\section{Introduction}

One of the main causes of training in superconducting magnets is thought to be frictional heating due to wire motion. 1,2 This is particularly true for particle accelerator magnets which use Rutherford-type conductors, where several strands are twisted together with a compaction factor of about $90 \%$. This leaves $10 \%$ of unfilled space, with many interfaces. The most cornmon technique to assemble a dipole or a quadrupole magnet is to clamp the superconducting coil into a very rigid structure (e.g., stainless steel collars) with a large compressive load (typically: $100 \mathrm{MPa}$ at room temperature). The coil precompression results in large frictional forces between the strands themselves and between the conductor and the conductor insulation, which should prevent any motion. However, during excitation, the strands are subjected to strong Lorentz lorces, which induce shear stresses at the metal/metal and metal/insulation interfaces. It can happen that the shear stress locally overcomes the frictional force leading to microslips or even stick-slip motions of the conductor strands. ${ }^{3-6}$ Also, the most favored insulator in superconducting magnet coils is Kapton, which has a relatively low yield point $(70 \mathrm{MPa}$ at $3 \%$ elongation and room temperature). Given the large stresses existing in the coil, plastic flows are thus likely to occur, especially
\end{abstract}

Table 1. Parameters of the Conductor for the TRISTAN Insertion Quadrupole Magnets

$\begin{array}{ll}\text { Height (mm) } & 9.09 \pm 0.03 \\ \text { Small width (mm) } & 1.19 \pm 0.01 \\ \text { Large width }(\mathrm{mm}) & 1.35 \pm 0.01 \\ \text { Twist pitch }(\mathrm{mm}) & 70 \pm 5 \\ \text { Number of strands } & 27 \\ \text { Strand diameter }(\mathrm{mm}) & 0.683 \\ \text { Filament diameter }(\mu \mathrm{m}) & 8.7 \\ \text { Copper to niobium-titanium rutio } & 1.7 \\ \text { Copper RRR } & 180 \pm 20 \\ \text { Critical current at 5 T and 4.2 K (A) } & 8160 \\ \text { N-value at 5 T } & 25\end{array}$

\footnotetext{
t Operated by the Universitics Rescarch Association, Inc., for the United States Department of Energy.
}

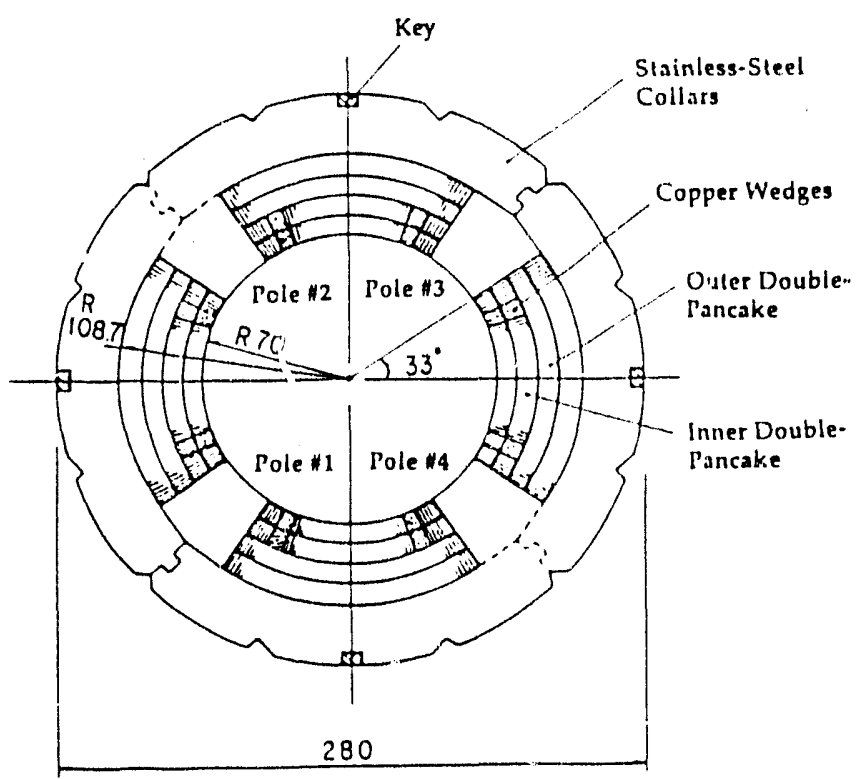

Figure 1: Cross-sectional view of the collared-coil assembly of the TRISTAN insertion quadrupcle magnels.

during excitation, also leading to conductor motions. These microslip, stick-slip, or plastic flow events will oi course produce a dissipation of energy which can be large enough to trigger a quench. $4,7,8$

The sudden motion of a wire is accompanied by two electromagnetic effects: an induced emf along the moved wire, and a change of flux caused by the minute dislocation of current. These iwo effects generate spikes in the coil voltage. The flux change is of course seen by the whole coil, but the induced emf only appears a'ong the moved wire. If, by some means, we can separate these two effects to only retain the induced emf, we should be able, from its amplitude, to estimate the amplitude of the wire movement and the order of magnitude of the energy release. This paper presents a measuring technique that was developed in order to separate these two effects and to enable direct buservation of the induced emf. We shall first describe the principles of the measuring technique. We shall then report wire-motion data from the excitation test of a superconducting quadrupole magnet. And last, we shall brieny discuss these data in relation to the stability theory.

The measurements presented here were carried out on one of the eight industrially-fabricatec quadrupole magnets for use in the TRISTAN low-beta insertions. The features of the ie iron-free magnets can be found elsewhere. ${ }^{9-11}$ Figure 1 shows a cross-sectional view of the collared-coil assembly; the four-layer $\cos 2 \theta$ coil consists of eight double-pancakes and was equipped with a total of 9 voltage taps, allowing voltage readout across each double-pancake. The parameters of the conductor are given in table 1. 


\section{Measuring Technicus}

Let us consider a wire of length / and cross-sertional area $A$ which carries a current density / in a magnetic flux densily $B$. If the wire moves a distance $d x$ ciuring a time $\mathrm{d} t$, a voltage $V_{\mathrm{em}}$ is induced along the wire. It is given by

$$
V_{\text {entf }}=B / \frac{\mathrm{d} x}{\mathrm{~d} I} \text {. }
$$

On the other hand, the work of the Lorentz force $F$ applied on the wire is

$$
\mathrm{d} W=F \mathrm{~d} x,
$$

where

$$
F=B \| A \text {. }
$$

By combining Eqs (1), (2), and (3), it comes that the energy $d E$ dissipated per unit cross-section of wire is

$$
\mathrm{d} E=V_{\mathrm{emf}} / \mathrm{dt} \text {. }
$$

Integrating Eq. (4) over the duration of the wire motion gives the total energy, $E$, released by the disturbance.

This simple model shows that if we can measure the induced emf we can estimate the energy dissipated by the wire motion.

As we described earlier, the wire motion is accompanied by a second electromagnetic effect: a change of nux caused by a minute dislocation of current. Practically, the voltages we measure are across large sections of coil. Therfore, they not only include the emf induced by the wire motion but also all the inductive voltages generated by fux changes across the coil. Among these flux changes is the one resulting from the minute dislocation of current. Another one is that resulting from the ramping of the current during the coil excitation. To access the emf we therefore need to eliminate these other components.

The coil in figure 1 is divided into 8 sections by mean of voltage taps. Let $V_{1}$ designate the voltage across the doublepancake where the wire motion orcurs, and let $V_{2}$ designate the voltage across the other dnuble-pancake of the same pole. These voltages are given by

$$
\begin{aligned}
& V_{1}=L_{1} \frac{\mathrm{d} l}{\mathrm{~d} t}+\frac{\mathrm{d} M_{\mathrm{ml}}}{\mathrm{d} t} \| A+V_{\mathrm{em} t}, \\
& V_{2}=L_{2} \frac{\mathrm{d} l}{\mathrm{~d} t}+\frac{\mathrm{d} M_{\mathrm{m} 2}}{\mathrm{~d} t} \| A,
\end{aligned}
$$

where $L_{i}$ are the self-inductances of the double pancakes, $M_{m i}$ are the mutual inductances betreen the double-pancakes and the moved wire, and $I$ is the current in the coil.

Now, the two double-pancakes we consider are of the same shape and are atop of each other, they thus embrace a very similar flux. Let $k$ designate the ratio of the two self-inductances (which only depends on the coil geometry)

$$
k=\frac{L_{2}}{L_{1}} \text {. }
$$

It seems legitimate to assume that the mutual inductances are also in the same ratio

$$
M_{2}=k M_{1} \text {, }
$$

Let us finally consider the canceled voltage $V_{\text {can }}$ defined by

$$
V_{\text {can }}=V_{1}-\frac{V_{2}}{k} \text {. }
$$

By combining Eqs. (5) through (9), it comes

$$
V_{\text {can }}-V_{\text {emf }} \text {. }
$$

Canceling the voltages across the two double pancakes of each pole by the ratio of the self inductances should therefore give us direct access to the emf generated by an eventual wire motion.

The wire tends of course to move in the direction of the Lorentz force; the sign of the emf tends therefore to be positive. It follows that the sign of the spike in the cancel signal indicates in which pancake the motion occurred.

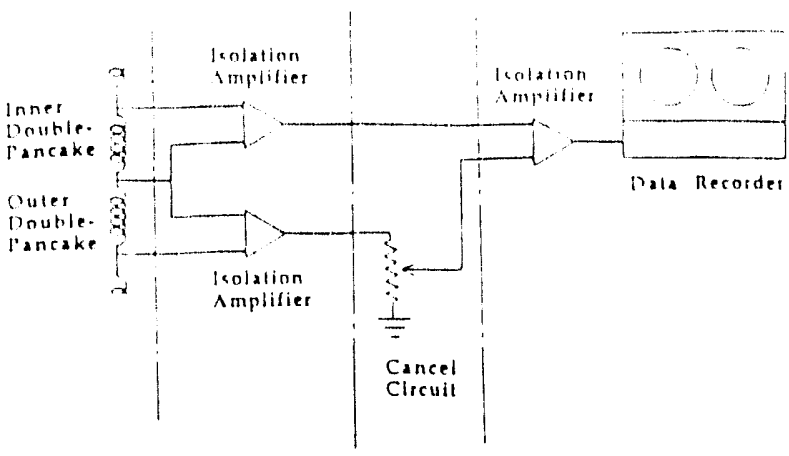

Figure 2: Schematic of the wire-motion readout system.

\section{Experimental Data}

The measurements were made on the insertion quadrupole magnet for TRISTAN described earlier. We shall refer to the four poles of the coil by the numbers 1 to 4 . Each pole has two double-pancakes, that we shall call respectively inner and outer. Four canceled voltages are considered, obtained from the difference between the inner and outer pancakes of each pole.

The measurement scheme is shown in figure 2. Each inner. and outer-pancake voltage enters an isolation amplifier and is plugged into the cancel circuit. The output of the cancel circuit goes through another isolation amplifiers and is finally recorded in a multi-channel analog data recorder. In the cancel circuit, the outer-pancake signal is attenuated by some factor and is subtracted from the inner-pancake signai. The attenuatior factor is deternined prior to the excitation test by subjecting the coil to an AC signal and canceling the voltages of each individual pole. The bandwidth of the isolation amplifiers is $100 \mathrm{KHz}$ and that of the data recorder is $20 \mathrm{KHz}$.

The energy released by the wire motion is calculated by inlegrating the voltage spike over the duration of the transitory times the current density, as indicated in Eq. (4). As mentioned above, the polarity of the spicte indicates the location of the wire motion. In our case, a positive spice indicates a motion that originated in the inner pancake, while a negative spike indlcates a motion that took place in the outer pancake. For a wire motion originating in the outes pancake, the energy caiculated from Eq. (4) is then divided by the cancelation factor to account for the attenuation.

While ramping the magnet current, many spikes were observed in all four canceled yoltages. Most of these spikes were not followed by a quench, and the vollage went back to zero. On the other hand, a large majority of the quenches were preceded by a spike, the initial polarity of which corresponded to that of the quenching pancake. Figure 3 shows two typical events that were recorded during the same magnet excitation. The event in figure $3 a$ originated at $3600 \wedge$ in the pole $\# 2$ outer pancake and is not followed by a quench. The event in figure $3 \mathrm{~b}$ took place in the pole \#3 inner pancake at $3750 \wedge$ and is followed by a gradual increase of the vollage, indicative of a quench. It is remarkable that not all the spikes are followed by a quench, but the fact that most of the quenches are preceded by one provides some evidence that spikes and quenches are correlated. The interpretation of these spikes as wire-motion induced signals is supported by similar ouservations that were made in previnus works, ${ }^{1,8,9}$ in coincidence with bursts of acoustic emission.

A particularity of the voltages shown in figure 3 is the structure of the transitory. It starts with a large spike, of durition $t$, followed by a damped oscillation. The canceled voltages of the other poles remain flat during the initial spike, but exhibit small fluctuations during the damped oscillations. $A$ plausible explanation is as follows. First, a wire rnoves suddenly, inducing an emf which is only seen by the canceled voltage of the 

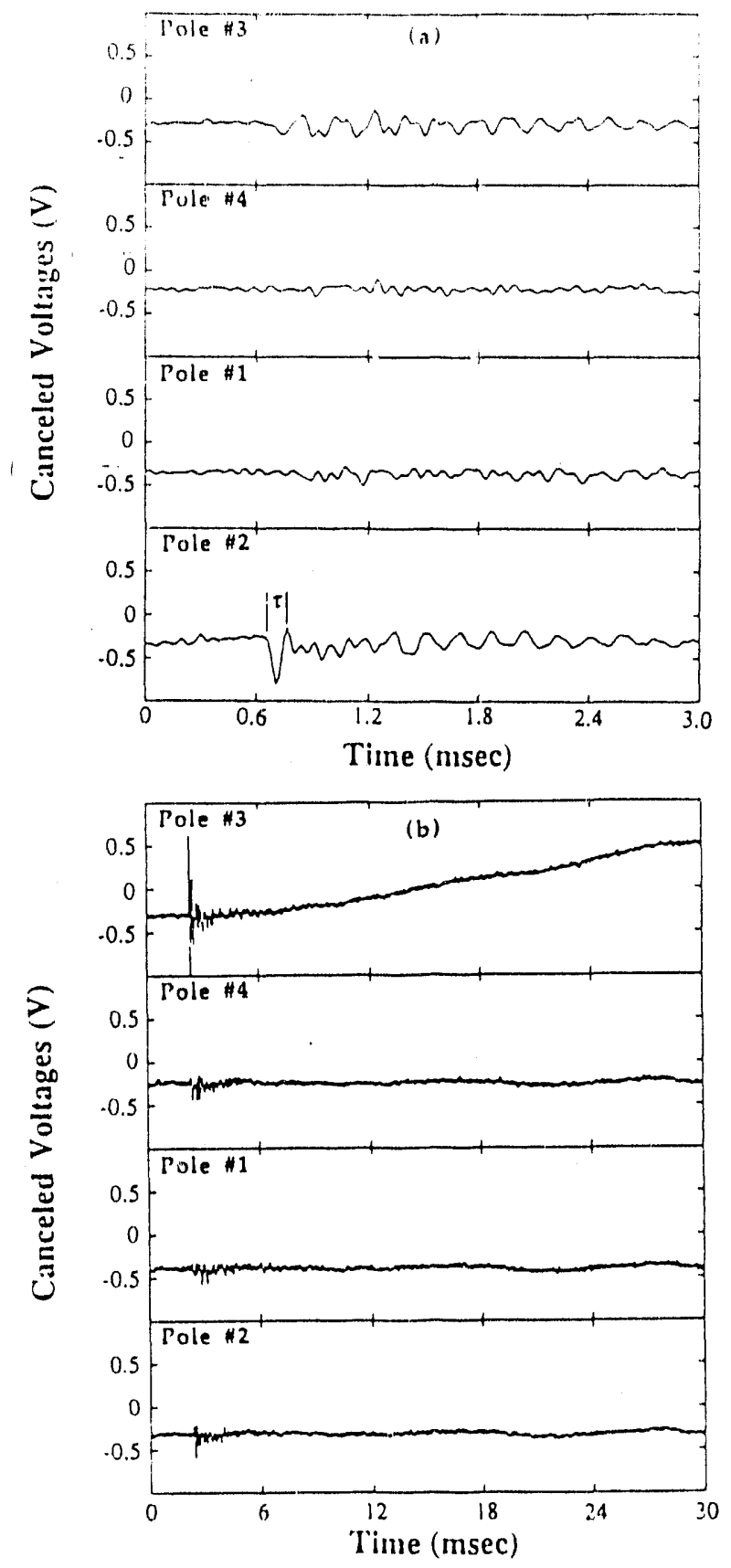

Figure 3: Example of wire-motion signals during the 8th excitation of a TRISTAN insertion quadrupole magnet. (a) AI $3600 \mathrm{~A}$. (b) at $3750 \mathrm{~A}$.

pcle where the motion occurs. The wire then hits its neighbors, inducing a mechanical vibration of the whole coil. The vibration is seen predominantly at the pole where it originated, but it is also picked up at the other poles, as observed in our signals. When calculating the energy released by the wire molion, the integration should therefore be limited over the time $\tau$. The order of magnitude of $\tau$ is typically $100 \mu \mathrm{s}$.

Figure 4 presents a plot of the energy release per unit cross section as a function of current for the wire-motion events of the second excitation of the magnet. Figure 5 presents the same plot for the eighth excitation. Both excitations lead to a training quench ( $3623 \mathrm{~A}$ for the 2 nd one, $3750 \mathrm{~A}$ for the 8 th one.) The wire-motion events occurred predominantly in the pole $\# 2$ outer pancake, but the 2 nd quench originated in the pole $\$ 2$ inner pancake, and the 8 th quench, in the pole $\# 3$ outer pancake.
An interesting feature of these two plots is that if we remow? the events taking place in the pole $\# 2$ outer-pancike, some events remain that took place in other pancakes, but the level of current at which they occur is differerent for the two excitations: for the 2 nd excitation, they occur all over the map, starting at low currents, but, for the 81 th excitation, nothing happens until we reach a level of current higher than that of the previous quenches. It is as if the sources of wire notion had been eliminated by the previous excitations, as predicted by the training model Another remarkable feature of these plots is tha: there is no clear trend between the energy release and the current, and the fact that the energy release of the event preceding the quench is not any larger than the previous ones. Of course, the calculation of the energy is not very accurate, for the duration of the spikes is not well defined, and their amplitudes may be affected by the small bandwidth of the analog recorder, but the statistical accumulation of events should reduce these uncertainties. This tells us that only a fraction of the energy release is deposited onto the conductor, eventually leading to a quench, and that this fraction is highly variable from one event to the other, probably depending on the cooling condition of the moved wire. In the following, we shall retain that the order of magnitude of the energy release, $E$, is $1 \mathrm{~mJ} / \mathrm{mm}^{2}$.

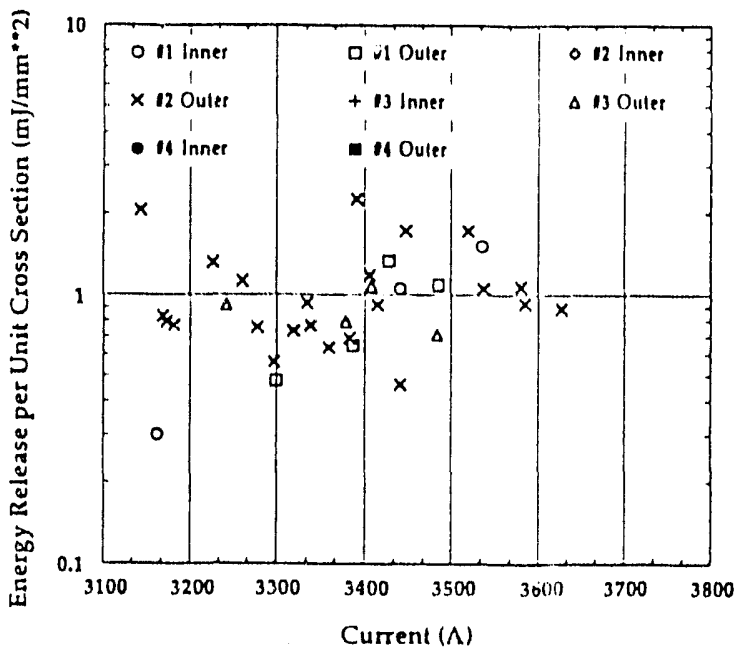

Figure 4: Energy release by wire motion as a function of current during the 2 nd excitation of a TRISTAN insertion quadrupole magnet.

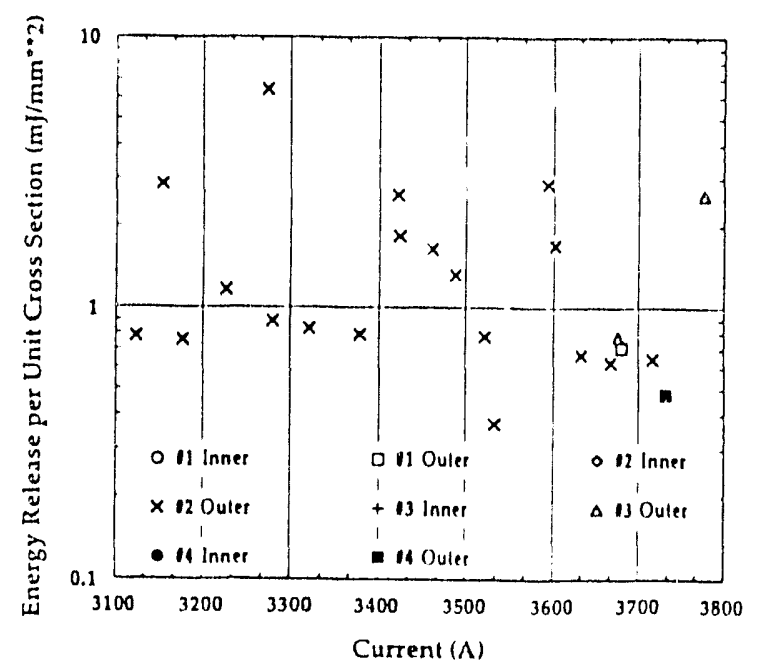

Figure 5: Energy relcase by wire motion as a function of current during the 8 ih Excitation of a TRISTAN insertion quadrupole magnet. 


\section{Discussion}

There have been many attempts to calculate the stability of superconducting magnets against local disturbances. $2.12-14$ This is indeed a difficult problem for it includes many variables. One must correctly model the transition from the superconducting to the normal-resistive state (taking into account the $\mathrm{N}$ value of the condcutor and the current redistribution through the copper matrix); one must also use adequate transient heattransfer coefficients to the helium. This is not the place to carry out a detailed analysis and we shall limit ourselves in determining the order of magnitude of the quantities here involved to verify that the values we measured make sense.

The energy releases of figures 4 and 5 are given per unit cross-section of wire. In a composite structure as that of a Rutherford-type conductor, the smallest part that can move is a strand. Also, because of the twisting, the maximum length of strand that can move is that corresponding to a half pitch. Let us thus assume that a half pitch of strand is moving. The total energy $Q$ released by the motion is

$$
Q=E \frac{\pi d^{2}}{4} \text {. }
$$

where $d$ is the strand diameter. With $E=1 \mathrm{~mJ} / \mathrm{mm}^{2}$, it comes

$$
Q=400 \mu \mathrm{J}
$$

The next step is to compare $Q$ with the energy $W_{C I}$ needed to heat-up the moved strand from the bath temperature $T_{0}$ to the critical temperature at the given current $T_{\mathrm{Cl}}$. We have

$$
W_{\mathrm{Cl}}=\left[H\left(T_{\mathrm{Cl}}\right)-H\left(T_{0}\right)\right] \frac{\pi d^{2}}{4} \frac{p}{2},
$$

where $H$ is the enthalpy per unit volume of conductor, and $p$ is the twist pitch. At $3500 \mathrm{~A}\left(B=6.2 \mathrm{~T}\right.$ and $\left.T_{\mathrm{Cl}}=5.45 \mathrm{~K}\right)$, it comes

$W_{\mathrm{CI}}=70 \mu \mathrm{J}$

As we said earlier, only a small fraction of the released energy has to be deposited onto the moved wire to induce a transition.

The last step is to evaluate how much of the energy release can be taken away by the helium. It is given by

$$
G_{\mathrm{He}}=\int_{0}^{\tau} \mathrm{d} t q(t) f \pi d t,
$$

where $q$ is the heat flux to the helium and $f$ is the fraction of the strand perimeter in contact with the helium. In the case of pool boiling helium, it is usually assumed that the cooling is only ef ficient as long it stays in the nucleate boiling regime, and the maximum heat flux that can be transferred during a transient is calculated as being that required to trigger the film boiling regime. ${ }^{12}$ For a transient of $100-\mu$ s duration, we have ${ }^{15}$

$$
\int_{0}^{\tau} \mathrm{d} t q(\mathrm{t})=5 \mathrm{~J} / \mathrm{m}^{2},
$$

and thus

$$
G_{\mathrm{He}}=\{400 \mu \mathrm{J}
$$

Depending on $f$, the helium has thus the capability to take away most of the heat released by the strand motion.

This very crude model shows that, on one side, we have a large energy dissipation, on the other side, we have $\rightarrow$ large cooling capability, and it is the difference between these two large terms that is deposited onto the wire and eventually heats it up. Depending on the local wet perimeter, the residual energy can be large enough to overcome the limited enthalpy of the wire and trigger a quench.

\section{Conclusion}

A technique was developed which allows the direst observation of the emf induced by wire motion and the calculation of the energy release resulting from the motion. Measurements were made on a superconducting quadrupole magnet, which showed that the excitation is accompanied by a great number of wire-motion events, of typical duration $100 \mu \mathrm{s}$, and typical energy release per unit cross-section of $1 \mathrm{~m} / / \mathrm{mm}^{2}$. A simple stability model, based on the motion of a ingle strand over a half twist pitch was then developed. which could account for the order of magnitude of the energies involved.

\section{Acknowledgements}

We are grateful to $Y$. Morita and $N$. Ohuchi for their help during the lest of the magnet.

\section{REFERF NCES}

[1] Y. Iwasa, "Conductor Motion in the Superconducting Magnet - a Review," l'roceedings of the Workshop on Stability of Superconductors in Helium I and II, Saclay, France, November 16-19, 1981 pp. 125-137, edited by Institut International du froid (Commision A1/2), Paris, France.

(2) A. Devred, "Quench Origins," to appear in the proceedings of 1989/1990 US Particle Accelerator School, Upton, NY, USA, July 29-August 4, 1990.

[3] R. S. Kensley, H. Maeda, and T. Iwasa, "Transient Slip Behavior of metal/insulator pairs at $4.2 \mathrm{~K}$," Cryogenics, 21 , pp. 479-489, 1981

[4] H. Maeda, O. Tsukamoto, and Y. Iwasa, "The Mechanism of frictional motion and its effects at $4.2 \mathrm{~K}$ in superconducting winding models," Cryogenics, 22, pp. 287. 295,1982

[5] T. Nishiura, S. Owaki, et al, "Frictional behavior in superconducting coil," IEEE Transaction on Magnetics, 21(2), pp. 384-387, March 1987.

[6] T. Takao, S. Honjo, and O. Tsukamoto, "Frictional Motion of Conductor in Superconducting Magnet and Quantification of Disturbance Energy, Ad. Cryo. Eng, 35, pp. 737-744, 1990.

[7] O. Tsukamoto, H. Maeda, and Y. Iwasa, "MicroslipInduced Degradation in a Braided Superconductor," Appi. Phys. Lett. 39(11), pp. 918-920, 1981.

[8] O. Tsukamoto and Y. Iwasa, "AE Technology as a Diagnostic and Monitoring Technique for Superconducting Magnets," Progress in Acoustic Emission, Proceedings of the 6th Int. AE Symp. pp. 332-344, 1982.

[9] K. Tsuchiya, K. Hosoyama, et al, "A prototype superconducting insertion quadrupole magnet for TRISTAN," Adv. in Cryo. Eng., 31, pp. 173-180, 1986.

[10] K. Tsuchiya, N. Ohuchi, et al, "Superconducting Quadrupole Magnets for the TRISTAN Low-Beta Insertion," Proceedings of the 11 th International Conference on Magnet Technology pp. 171-175, 1990.

[11] K. Tsuchiya, K. Egawa, et al, "Performance of the Eight Supercondcuting Quadrupole Magnets for the Tristan LowBeta Insertions," in these proceedings.

[12] C. Schmidt, "Review of Steady State and Transient Heat Transfer in Pool Boiling Helium l," Proceedings of the Workshop on Stability of Superconductors in Helium 1 and II. Saclay, France, November16-19, 1981 pp. 17-31, edited by Institut International du froid (Commision A1/2), Paris, France.

[13! O. Tsukamoto, T. Takao, and S, Honjo, "Stability Analysis of Superconducting Magnet: an Approach to Quantification of Energy of Disturbance Caused by Condurtor Motion," Cryogenics, 29, pp. 616-620, 1989.

[14] L. Malinowski, "Analytical Method for calculation of Critical Energy of Technical Superconductors Taking into Account Transient Heat Transfer," Cryogenics, 30, pp. 27-31, 1990.

[15] M. N. Wilson, Superconducting Magnets, Clarendon Press, Oxford, p. 114, 1983. 

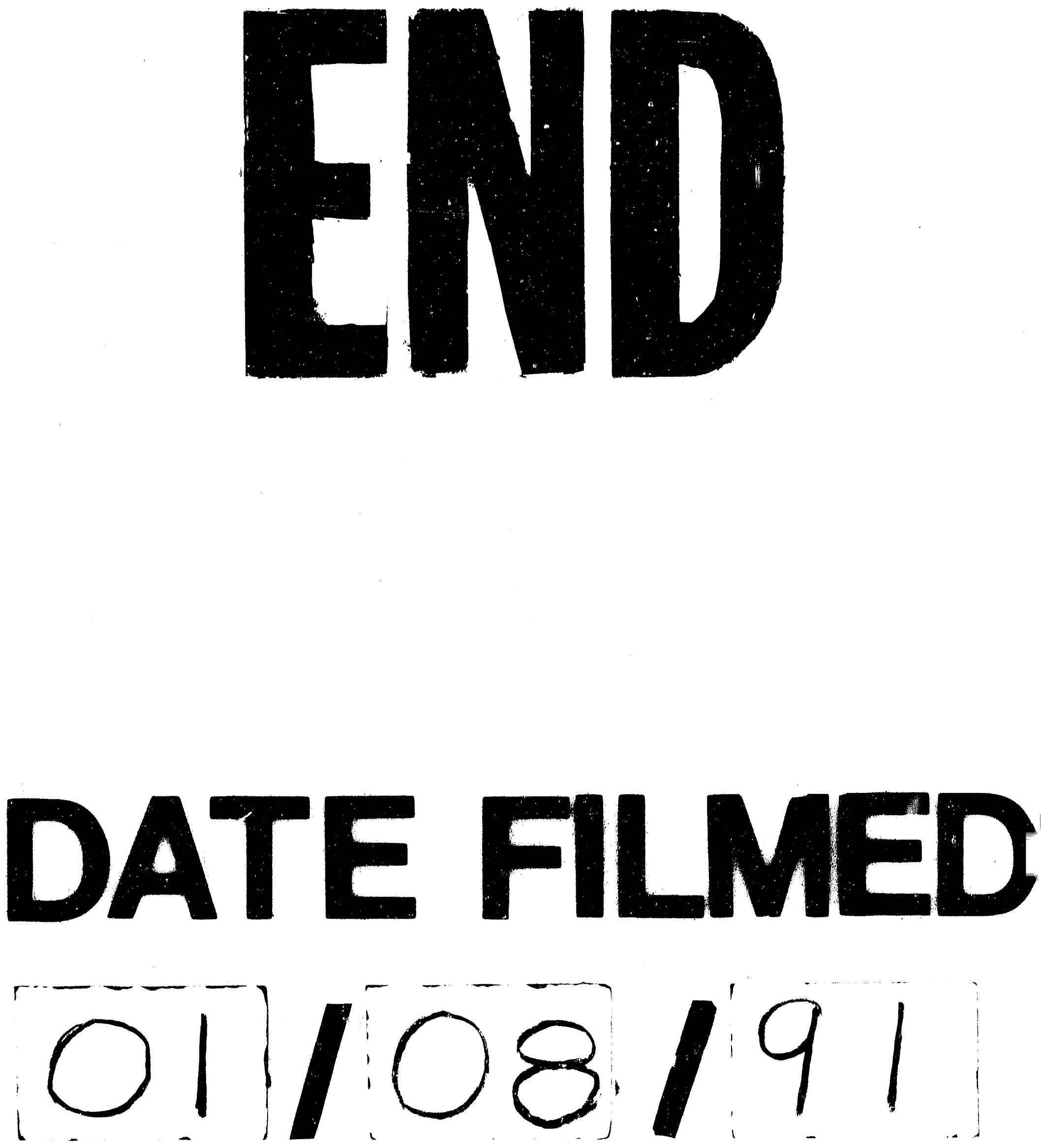
